\title{
Emery-Dreifuss muscular dystrophy as a possible cause of coronary embolism
}

\author{
Atsushi Tada ${ }^{1}$, Takao Konishi ${ }^{1}$, Takuma Sato ${ }^{1}$, Tomoya Sato ${ }^{1}$, Takuya Koizumi ${ }^{1}$, \\ Sakae Takenaka ${ }^{1}$, Yoshifumi Mizuguchi ${ }^{1}$, Takahide Kadosaka ${ }^{1}$, Ko Motoi ${ }^{1}$, \\ Yuta Kobayashi ${ }^{1}$, Hirokazu Komoriyama ${ }^{1}$, Yoshiya Kato ${ }^{1}$, Kazunori Omote ${ }^{1}$, \\ Shingo Tsujinaga ${ }^{1}$, Rui Kamada ${ }^{1}$, Kiwamu Kamiya ${ }^{1}$, Hiroyuki Iwano ${ }^{1}$, \\ Toshiyuki Nagai ${ }^{1}$, Nanase Okazaki ${ }^{2}$, Yoshihiro Matsuno ${ }^{2}$, Toshihisa Anzai ${ }^{1}$ \\ ${ }^{1}$ Department of Cardiovascular Medicine, Faculty of Medicine and Graduate School of Medicine, \\ Hokkaido University, Sapporo, Japan \\ ${ }^{2}$ Department of Surgical Pathology, Hokkaido University Hospital, Sapporo, Japan
}

A 43-year-old man, who had a history of atrial arrhythmia and anterior myocardial infarction due to coronary embolism presented to the cardiology clinic for further examination. He was previously diagnosed as Emery-Dreifuss muscular dystrophy (EDMD) involving skeletal muscles in the lower limbs. An electrocardiogram showed junctional rhythm at a heart rate of $39 \mathrm{bpm}$ with atrial arrest and poor $r$ progression (Fig. 1A). Echocardiography revealed a left ventricular dilatation and a diffuse hypokinesis of the left ventricle with ejection fraction of $48 \%$ (Fig. 1B). Cardiac magnetic resonance imaging demonstrated a subendocardial late gadolinium enhancement at the anterior wall of left ventricle (Fig. 1C), consistent with the old anterior myocardial infarction. Right ventricular endomyocardial biopsy revealed irregular sizes of nuclei with vacuolation and reduced myocardial fibers (Fig. 1D), whereas interstitial fibrosis was not apparent (Fig. 1E). In EDMD, fibrosis or adipose tissue replacement usually starts in the atria, leading to atrial arrhythmias, and then affects the ventricles. This case suggests that EDMD in the early-stage does not necessarily display apparent fibrosis, despite the presence of mild myocardial abnormalities in histopathology. Furthermore, EDMD might be one of the possible causes of early-onset atrial arrhythmias causing coronary artery embolism or embolic cerebral infarction. He underwent cardiac resynchronization therapy defibrillator because he had symptomatic bradycardia and a history of ventricular tachycardia. At the 5-month follow-up, the patient remained well.

Conflict of interest: None declared

Address for correspondence: Takao Konishi, MD, PhD, Department of Cardiovascular Medicine, Faculty of Medicine and Graduate School of Medicine, Hokkaido University, West 7, North 15, Kita-ku, Sapporo, 060-8638, Japan, tel: +81-11-706-6973, fax: +81-11-706-7874, e-mail: takaokonishi0915@gmail.com 

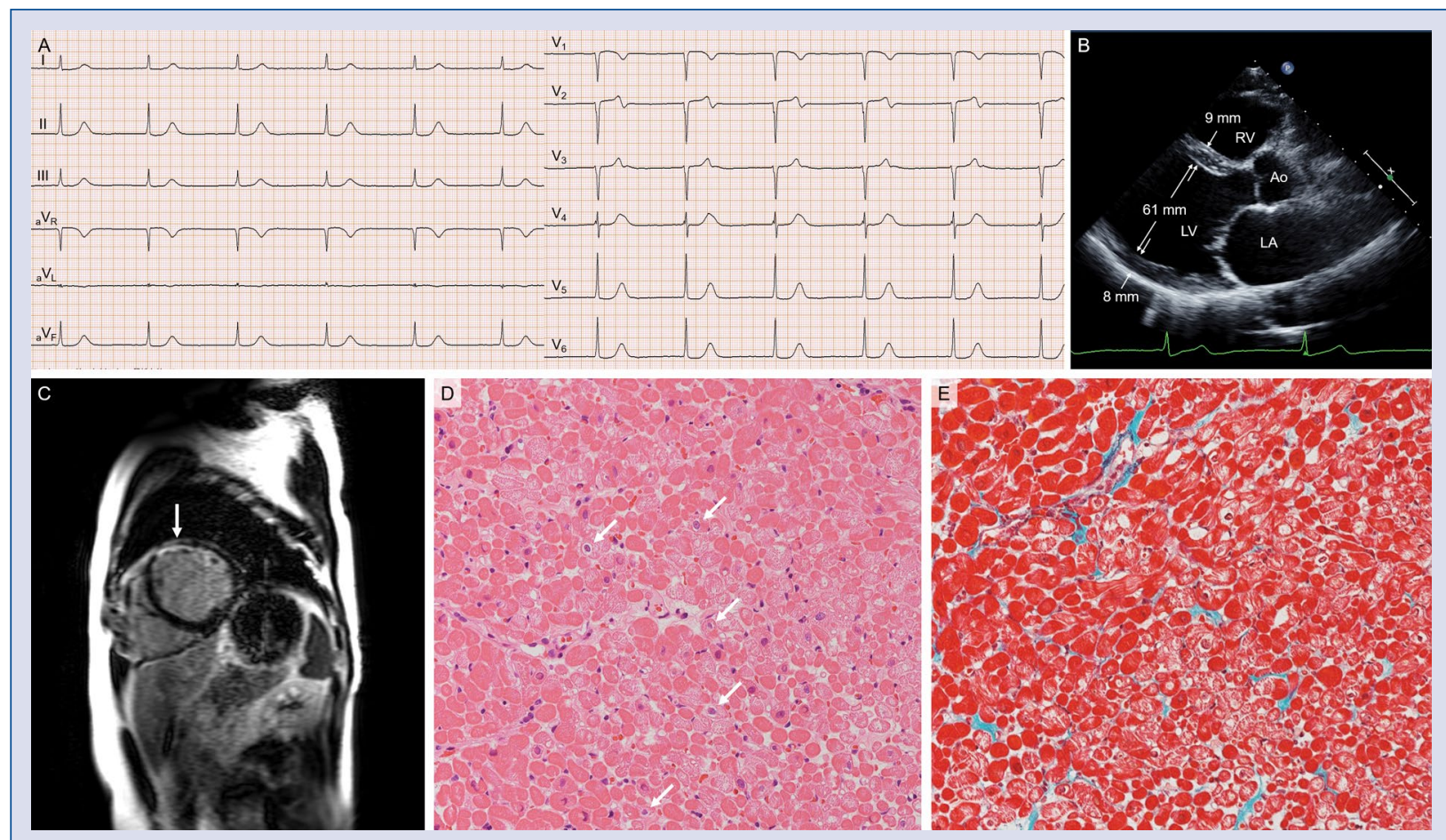

Figure 1. A. A 12-lead electrocardiogram showed atrial arrest with junctional rhythm; B. Echocardiogram (parasternal long axis) showing left ventricle (LV) dilatation; LA — left atrium; RV — right ventricle; Ao — aorta; C. Cardiac magnetic resonance imaging showing a late gadolinium enhancement (arrow); D. Histopathology after hematoxylin and eosin staining showed irregular sizes of nuclei with vacuolation (arrows) and reduced myocardial fibers; E. Slight interstitial fibrosis was stained green after elastica-Masson staining. 